\title{
Radiculopathy caused by lumbar epidural varix
}

Jiyong Shin, $\mathrm{MD}^{1}$; Nawon $\mathrm{Oh}^{2}$; Jisoon Huh, $\mathrm{MD}^{3}$; Chang Lim Hyun, MD, $\mathrm{PhD}^{4}$; Joong-Goo Kim, MD'; Sa-Yoon Kang, MD'; Jung-Hwan Oh, MD'

'Department of Neurology, Jeju National University School of Medicine, Jeju, Republic of Korea

${ }^{2}$ Jeju National University School of Medicine, Jeju, Republic of Korea

${ }^{3}$ Department of Neurosurgery, Jeju National University School of Medicine, Jeju, Republic of Korea

${ }^{4}$ Department of Pathology, Jeju National University School of Medicine, Jeju, Republic of Korea

\section{Journal of Neurocritical}

\section{Care}

\section{CASE REPORT}

Received: October 28, 2020

Revised: December 21, 2020

Accepted: December 23, 2020

Corresponding Author:

Jung-Hwan Oh, MD

Department of Neurology, Jeju National University School of Medicine, 15 Aran

13-gil, Jeju 63241, Korea

Tel: +82-64-717-1620

Fax: +82-64-717-1320

E-mail: oh.junghwan77@gmail.com

Background: Lumbar epidural varix (LEV) is a very rare condition caused by dilatation of the vertebral venous plexus. LEV can result in lumbosacral radiculopathy, which is often mistaken for lumbosacral herniated intervertebral disc (HIVD).

Case Report: A 72-year-old man visited the emergency department (ED) with radiating pain of the right leg that had developed 3 weeks previously. Before the ED visit, he was diagnosed with lumbosacral radiculopathy due to HIVD based on lumbar X-rays at an outpatient clinic. Despite conservative treatment, his symptoms deteriorated. On magnetic resonance imaging at the $E D$, an epidural cystic mass in the right L5-S1 with multiple HIVDs was observed. The mass was surgically removed, and the histological findings showed a dilated vessel with a thrombus, which led to the final diagnosis of LEV.

Conclusion: In lumbosacral radiculopathy, LEV should be considered as a possibility even if degenerative spine disease is observed on lumbar X-rays.

Keywords: Radiculopathy; Spine; Varicose veins

\section{INTRODUCTION}

Lumbar epidural varix (LEV) is a very rare condition caused by dilation of the internal vertebral venous plexus, and can result in lumbosacral radiculopathy by irritating or compressing the epidural or intervertebral foramen [1]. According to previous studies, $0.067 \%$ to $1.2 \%$ of patients with lower back pain or leg paresthesia are diagnosed with LEV [2]. Clinically, LEV is often confused with lumbosacral herniated intervertebral disc (HIVD), a common cause of lumbosacral radiculopathy, and it is usually diagnosed during or after surgery [3]. Here, we report a rare case of a patient diagnosed with LEV who presented with lumbosacral radiculopathy.

\section{CASE REPORT}

A 72-year-old man with past history of hypertension, diabetes mellitus, and hyperlipidemia visited the emergency department (ED) of Jeju National University Hospital with a complaint of radiating pain of the right leg that had developed 3 weeks previously. $\mathrm{He}$ visited an outpatient clinic 10 days before presenting to the ED. At that time, lumbar X-rays showed compression fractures in

(C) 2020 The Korean Neurocritical Care Society

This is an Open Access article distributed under the terms of the Creative Commons Attribution Non-Commercial License (http://creativecommons.org/licenses/by-nc/4.0/) which permits unrestricted noncommercial use, distribution, and reproduction in any medium, provided the original work is properly cited. 
the T12 and L2 vertebral bodies and L4-5 disc space narrowing on lumbar X-rays, and the patient was diagnosed with lumbosacral radiculopathy due to HIVD. He was prescribed nonsteroidal anti-inflammatory drugs and underwent a nerve block injection, but his symptoms steadily worsened. On the day of the patient's ED visit, his symptoms were so severe that he could not walk.

The patient also stated that he had suffered from lower back pain for 1 month and reported no history of urinary or fecal incontinence, febrile sensation, or rash. He reported no history of trauma or intense exercise. A neurologic examination at the ED showed that his muscle power had been reduced to Medical Research Council grade 4 for right big toe extension. A sensory deficit was documented over the right L4 to S1 dermatome levels. The deep tendon reflex was hypoactive on the right knee and ankle, and Babinski reflexes were negative. In the complete blood count, the levels of white blood cells, platelets, and hemoglobin were $11,700 / \mathrm{mm}^{3}$ (normal range, $4,000-10,000 / \mathrm{mm}^{3}$ ), $385,000 / \mathrm{mm}^{3}$ (normal range, $150,000-450,000 / \mathrm{mm}^{3}$ ), and 12.6 $\mathrm{g} / \mathrm{dL}$ (normal range, 13-17 g/dL), respectively. In the coagulation test, the prothrombin time-international normalized ratio and activated partial thromboplastin time were 0.98 (normal range, 0.88-1.20) and 26 seconds (normal range, 20.0-36.0 seconds), respectively: both values were within the normal range. Lumbar magnetic resonance imaging (MRI) showed multiple bulging intervertebral discs. An epidural cystic mass measuring $18 \times 11 \times 19 \mathrm{~mm}$ was also observed in the right L5-S1 intervertebral foramen and the right epidural space. The mass presented as hyperintense on T1-weighted images, hypointense on T2-weighted images, and heterogeneously enhanced on T1 gadolinium-enhanced images (Fig. 1). Emergent partial hemilaminectomies were performed. The ligamentum flavum showed hypertrophy, had turned black, and compressed the right L5 root. A black cystic epidural mass was identified in the shoulder of the right $S 1$ root during surgery. The mass was grossly removed and was shown to be a dilated vessel wall with an organized thrombus on the histological examination (Fig. 2). Finally, the mass lesion was diagnosed as LEV.

The patient's symptoms gradually improved starting on the second day after surgery, Two weeks after surgery, although hypoesthesia in the sole of the right foot was observed, the radiating pain in the right leg had disappeared entirely, and the muscle power of the right big toe was also normal.

\section{DISCUSSION}

Our patient was initially diagnosed with lumbosacral radiculopathy caused by HIVD and conservatively treated at an outpatient

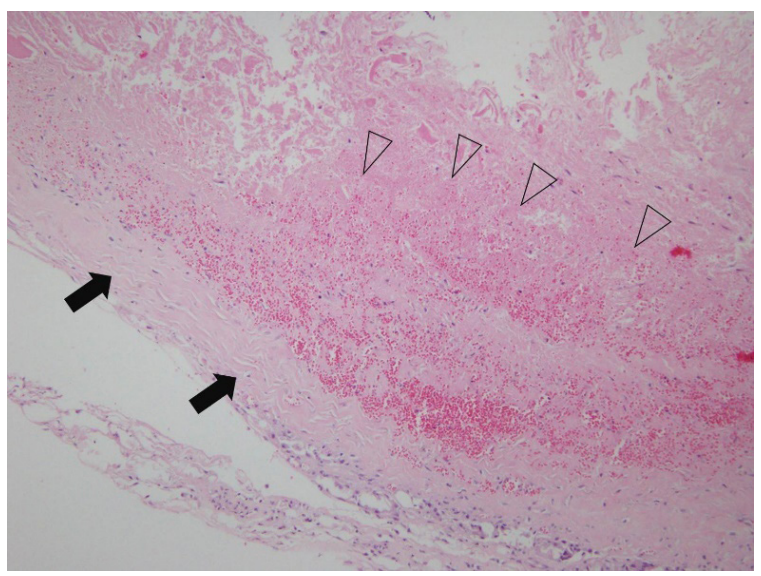

Fig. 2. Dilated and tortuous vessel wall (arrows) with an organized thrombus (arrowheads) in the histological examination (H\&E, $\times 100)$.
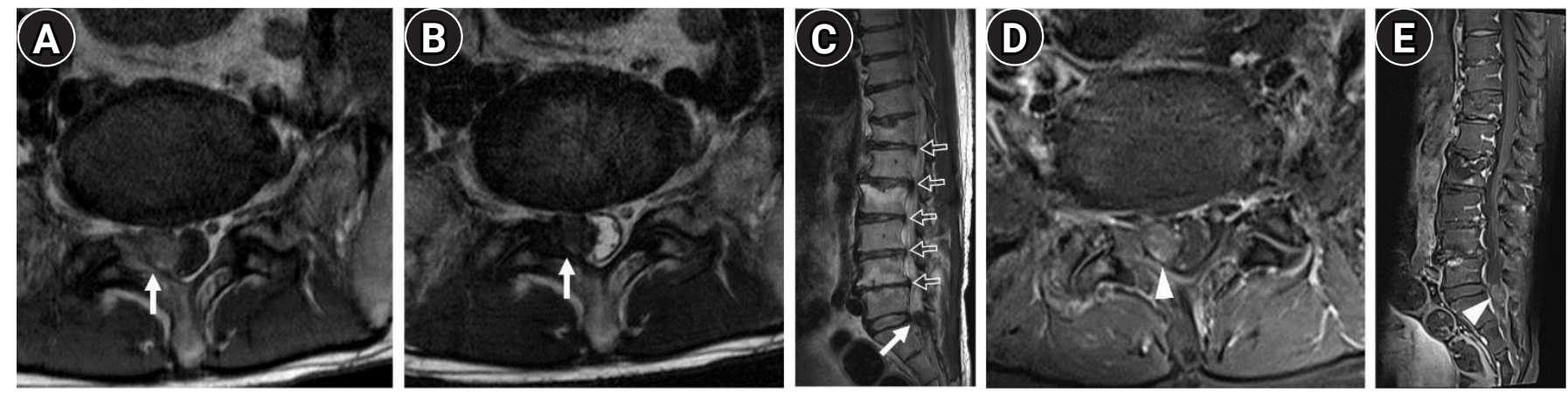

Fig. 1. Preoperative lumbar magnetic resonance images. Axial T1-weighted (A) and T2-weighted (B), and sagittal T2-weighted (C) images show an irregular mass lesion (closed arrows) involving the right facet joint at L5-S1 and the right epidural space at the L5-S1 level. Multiple bulging discs (open arrows) with compression fractures in the T12 and L2 vertebral bodies are also shown on sagittal T2-weighted images (C). Heterogeneous enhancement of the mass is shown on gadolinium-enhanced T1-weighted axial (D) and sagittal images (E) (arrowheads). 
clinic, but the symptoms worsened, prompting him to visit the ED. A mass lesion around the epidural space at L5-S1 with multiple spinal degenerative changes was observed on lumbar MRI performed at the ED. The mass was finally diagnosed as LEV after surgical removal.

LEV is challenging to diagnose because of clinicians' unfamiliarity with the disease and its variable findings on MRI, which are attributed to diversiform thrombosed or flowing blood states. Three core MRI findings have been proposed in the literature: an epidural mass of intermediate signal intensity, serpiginous dilated veins, and notable lumbar segmental veins [4]. Slin'ko and Al-Qashqish [1] described the MRI findings more specifically. According to those researchers, normal epidural veins are generally indistinguishable on MRI. Instead, the epidural varix can be visualized in the form of a signal void, or with moderate blood flow, the signal intensity is low on T1-weighted images. On T2-weighted images, the signal intensity is low with high blood flow and high with low blood flow. A pattern of spottiness is rarely seen in partial thrombosis of varicose veins. In some cases, the mass can be enhanced heterogeneously or intensively [1]. These various patterns of MRI findings are often confused with HIVD, synovial cyst, schwannoma, abscess, or a metastatic tumor [5]; therefore, LEV is often diagnosed during or after surgery.

If a clinician suspects LEV, venography can be performed in addition to MRI. If this test shows evidence that is strongly suggestive of LEV, the surgeon has microsurgical treatment options, such as whether to incompletely interrupt the blood flow related to the mass with regard to the collateral flow. Slin'ko and Al-Qashqish [1] suggested spinal venography as a helpful examination, using contrast injection through transdural or spinous process approaches. Based on computed tomography (CT), CT myelography, and MRI, the researchers performed venography in all cases that were assumed not to be typical disc herniation or spinal stenosis. However, only 51 out of 433 patients who underwent venography were identified as having LEV, and of those, 43 cases were surgically confirmed. However, selective spinal angiography is not helpful to identify epidural venous plexus.

The spinal epidural venous system is valveless, connecting the spinal venous flow with the inferior vena cava (IVC). It comprises internal and external venous networks. The internal vertebral venous system consists of anterior and posterior divisions. LEV is known to be associated with the anterior division since it lies before the posterior margins of the vertebral bodies and intervertebral discs [6]. The pathophysiology of LEV formation is not fully understood. Excessive intraabdominal pressure caused by pregnancy, an abdominal tumor, or trauma can keep spinal venous blood from returning to the heart through the IVC, resulting in engorgement of the valveless spinal venous plexus $[7,8]$. There have also been reports of LEV in patients with coagulopathy (e.g., protein C deficiency) or vasculitis (e.g., Behçet disease) [7].

Some researchers have proposed that degenerative spine diseases, including HIVD, may cause varix by directly irritating the venous endothelium $[1,9]$. Interestingly, it has been reported that 48 of 1,091 patients who underwent surgery for HIVD turned out to have LEV [10]. Therefore, it should be noted that although LEV can occur independently, it can also be accompanied by degenerative spine diseases such as HIVD, a common cause of lumbosacral radiculopathy. Unfortunately, blood tests for coagulopathy or vasculitis were not performed in our patient, but there was no condition that could increase intraabdominal pressure. However, it is cautiously assumed that the multiple HIVDs observed on lumbar $\mathrm{X}$-ray images may have caused LEV in this patient. As far as is known, the optimal treatment of LEV is surgical resection and decompression, although Tofuku et al. [11] published a case of lumbar LEV that spontaneously regressed in 2 weeks. Whether to remove all perilesional epidural veins is not well established.

In conclusion, LEV is easy to misdiagnose and underestimate. When physicians encounter patients with lumbosacral radiculopathy, they should always be mindful of the possibility of LEV, even if degenerative spine diseases such as HIVD are strongly assumed to be present based on lumbar X-rays. Lumbar X-rays can show bony structural abnormalities, but cannot be used to evaluate the condition of soft tissues around bones (e.g., blood vessels and nerves); therefore, X-ray examinations have low effectiveness in discriminating the cause of radiculopathy. Finally, the exact mechanisms involved in the pathogenesis of LEV remain unclear; systematic research is therefore needed to establish these mechanisms conclusively.

\section{ARTICLE INFORMATION}

\section{Ethics statement}

This study was conducted with permission from the Institutional Review Board and Ethics Committee of Jeju National University Hospital (IRB No. 2020-10-015). The informed consent was waived due to the retrospective nature of the study.

\section{Conflict of interest}

Dr. JH Oh is an editorial board member of the journal but did not involve in the peer reviewer selection, evaluation, or decision process of this article. No other potential conflicts of interest relevant to this article were reported. 


\section{ORCID}

Jiyong Shin

https://orcid.org/0000-0001-5852-4366

Nawon Oh

https://orcid.org/0000-0001-6762-0579

Jisoon Huh

https://orcid.org/0000-0001-6111-4296

Chang Lim Hyun

https://orcid.org/0000-0002-6740-1357

Joong-Goo Kim

Sa-Yoon Kang

Jung-Hwan Oh https://orcid.org/0000-0002-4166-6023

https://orcid.org/0000-0001-6755-089X

https://orcid.org/0000-0003-3997-3267

\section{Author contributions}

Conceptualization: JHO. Data curation: JS, CLH, JH. Formal analysis: JS, CLH, JH, NO, JHO. Investigation: JS, SYK, JGK. Supervision: JHO. Visualization: JS, JH, CLH. Writing-original draft, review, \& editing: JS, JHO.

\section{REFERENCES}

1. Slin'ko EI, Al-Qashqish II. Surgical treatment of lumbar epidural varices. J Neurosurg Spine 2006;5:414-23.

2. Endres S. Epidural varicosis as a possible cause of radicular pain: a case report. J Med Case Rep 2011;5:537.

3. Bursalı A, Akyoldas G, Guvenal AB, Yaman O. Lumbar epidural varix mimicking disc herniation. J Korean Neurosurg Soc 2016;59:410-3.

4. Choi S, Kim KY, Lee S, Yoon S. Efficacy of enhanced MRI in

epidural varix: report of six cases. J Korean Soc Spine Surg 2006;13:210-4.

5. Gálvez MM, Cordovez JM, Okuma CP, Montoya CM, Asahi TK. Diagnóstico diferencial de hernia discal. Rev Chil Radiologia 2017;23:66-76.

6. Zimmerman GA, Weingarten K, Lavyne MH. Symptomatic lumbar epidural varices: report of two cases. J Neurosurg 1994; 80:914-8.

7. Paksoy Y, Gormus N. Epidural venous plexus enlargements presenting with radiculopathy and back pain in patients with inferior vena cava obstruction or occlusion. Spine (Phila Pa 1976) 2004;29:2419-24.

8. Hirabayashi Y, Shimizu R, Fukuda H, Saitoh K, Igarashi T. Effects of the pregnant uterus on the extradural venous plexus in the supine and lateral positions, as determined by magnetic resonance imaging. BrJ Anaesth 1997;78:317-9.

9. Gundry CR, Heithoff KB. Epidural hematoma of the lumbar spine: 18 surgically confirmed cases. Radiology 1993;187:42731.

10. Gümbel U, Pia HW, Vogelsang H. Lumbosacral vascular anomalies as the cause of ischialgia. Acta Neurochir (Wien) 1969; 20:131-51.

11. Tofuku K, Koga H, Yone K, Komiya S. Spontaneous regression of symptomatic lumbar epidural varix: a case report. Spine (Phila Pa 1976) 2007;32:E147-9. 